\title{
Revisión
}

\section{Curvas presión-volumen en la lesión pulmonar aguda}

\author{
GUILLERMO M. ALBAICETA \\ Unidad de Cuidados Intensivos. Hospital Universitario Central de Asturias. Departamento de Biología Funcional. \\ Universidad de Oviedo. Oviedo. Asturias. España.
}

\begin{abstract}
Se ha estudiado ampliamente las curvas estáticas de presión-volumen del sistema respiratorio en pacientes con lesión pulmonar aguda como reflejo de los fenómenos de aireación y reclutamiento alveolar y como herramienta para el ajuste de la ventilación mecánica. Los puntos de inflexión de dichas curvas permiten identificar tanto las presiones en las que comienza y termina el reclutamiento como aquellas en las que comienza el desreclutamiento. Sin embargo, la selección de parámetros ventilatorios basada en estas curvas presenta varios problemas, derivados del hecho de que la selección de PEEP y presiones en meseta en estos pacientes debe balancearse entre los beneficios del reclutamiento y los riesgos de la sobredistensión producida por presiones elevadas. Está por ver si nuevos datos derivados de estas curvas permiten optimizar los parámetros de ventilación mecánica o predecir la respuesta de un paciente a un cambio en los ajustes del ventilador.
\end{abstract}

PALABRAS CLAVE: Curvas presión-volumen. Mecánica respiratoria. PEEP. Lesión inducida por la ventilación mecánica. Lesión pulmonar aguda.

\section{PRESSURE-VOLUME CURVES IN ACUTE PULMONARY INJURY}

Static pressure-volume curves of the respiratory system from patients with acute lung injury have been extensively studied as a marker of aeration and recruitment phenomena and as a tool to set mechanical ventilation. The inflection points of these curves allow to identify both the pressures in which recruitment starts and finishes

Correspondencia: Dr. G.M. Albaiceta.

Unidad de Cuidados Intensivos. Hospital Universitario Central de Asturias. Celestino Villamil, s/n. 33006 Oviedo. Asturias. España.

Correo electrónico: guillermo.muniz@sespa.princast.es

Manuscrito aceptado el 24-4-2008. and those in which derecruitment starts. However, setting the ventilatory parameters based on these curves has some problems, derived from the fact that setting PEEP and plateau pressures in these patients must balance between the benefits of recruitment and the risks of overstretching caused by high pressures. It remains to be determined if new data derived from these curves are useful to optimize ventilatory settings or to predict the response of a patient to a change in the ventilatory settings.

KEY WORDS: Pressure-volume curves. Respiratory mechanics. PEEP. Ventilator-induced injury. Acute pulmonary injury.

\section{INTRODUCCIÓN}

La ventilación mecánica es una medida de soporte esencial en pacientes con lesión pulmonar aguda y síndrome del distrés respiratorio agudo (SDRA). La aplicación de presión positiva en la vía aérea permite afrontar el aumento del trabajo respiratorio que supone la disminución de la elasticidad del sistema respiratorio causada por la respuesta inflamatoria, así como la reaireación de zonas de parénquima donde predomina el shunt (bien por colapso alveolar o por inundación del espacio aéreo) ${ }^{1}$. Sin embargo, esta misma presión positiva es capaz de producir lesión del parénquima pulmonar ${ }^{2}$. El estrés mecánico en el pulmón, además de producir una lesión directa del espacio aéreo y los vasos, induce una respuesta inflamatoria que puede perpetuar la lesión pulmonar ${ }^{3}$. Esta lesión asociada a la ventilación mecánica es un determinante de la mortalidad de los pacientes con SDRA, de tal manera que las únicas estrategias de ventilación que han demostrado disminuir la mortalidad en esta población de pacientes son las que se han dirigido a limitarla ${ }^{4-6}$. Los mecanismos de la lesión inducida por la ventilación mecánica o en relación 
con ésta se han revisado recientemente ${ }^{2,3,7}$, y su descripción excede los objetivos de este artículo.

La lesión relacionada con el ventilador, por lo tanto, comienza con un estímulo mecánico en el epitelio respiratorio, tanto a nivel de la vía aérea como del alveolo. Esta relación con factores mecánicos es causa del amplio interés que la mecánica respiratoria ha despertado como medio para optimizar la ventilación de la manera menos lesiva posible.

El estrés mecánico ejercido por la ventilación dependerá del estado mecánico inicial (el volumen teleespiratorio) y de la energía transmitida en cada ciclo respiratorio ${ }^{8,9}$. Las curvas estáticas de presiónvolumen -es decir, las que se obtienen en condiciones en que el componente resistivo de la presión es cero (o al menos despreciable)- permiten evaluar ambos factores. Aunque más adelante se verá que esta evaluación es solamente parcial, es una de las medidas más completas de la mecánica respiratoria que se puede obtener a la cabecera del paciente.

Teniendo en cuenta todo lo descrito hasta ahora, la curva estática de presión-volumen es una buena herramienta para el estudio de las propiedades mecánicas del sistema respiratorio y un medio prometedor para un ajuste de la ventilación mecánica dirigido a minimizar el estrés mecánico del parénquima pulmonar.

En esta revisión se discuten diferentes aspectos sobre el trazado y la interpretación de dichas curvas, así como su utilidad para la optimización de la ventilación en pacientes con lesión pulmonar aguda.

\section{¿QUÉ REPRESENTAN LAS CURVAS PRESIÓN-VOLUMEN?}

La curva estática de presión-volumen se obtiene mediante la representación gráfica del volumen pulmonar para una presión estática (es decir, en ausencia de flujo en la vía aérea) determinada. Al prescindir de este componente resistivo, la presión medida es la verdadera presión de retroceso elástico del parénquima pulmonar.

La histéresis del sistema respiratorio hace que la relación volumen-presión sea diferente durante la inspiración y la espiración. Esto origina las dos ramas de la curva, que se muestran en la figura 1A. La pendiente en cada punto de la curva equivale al cociente entre el incremento de volumen y el incremento de presión en ese punto. Como el lector habrá notado, dicho cociente es la distensibilidad del sistema respiratorio a ese volumen determinado.

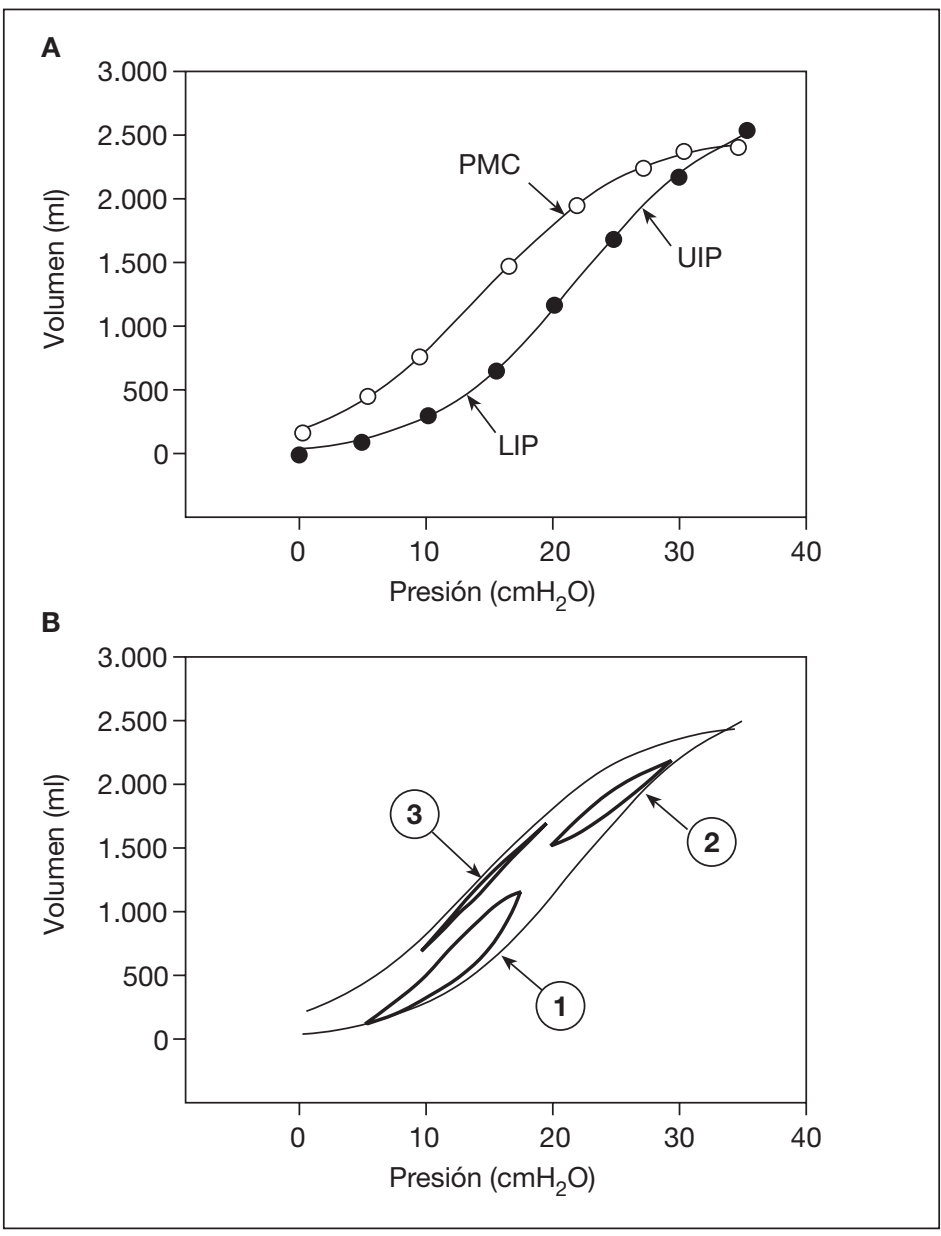

Figura 1. A: morfología característica de las ramas inspiratoria (puntos negros) y espiratoria (puntos blancos) de una curva estática de presión-volumen en un paciente con lesión pulmonar aguda, en la que se han señalado los puntos de inflexión (LIP. punto bajo inspiratorio; PMC: punto de máxima curvatura espiratoria; UIP: punto alto inspiratorio). B: sobre la misma curva se han inscrito tres ciclos tidales. Empezando desde una PEEP baja y un pulmón pobremente reclutado, el ciclo tidal se situa próximo a la rama inspiratoria, consiguiendo algo de reclutamiento al final de la inspiración (1). Al subir la PEEP a niveles altos, el ciclo se desplaza hacia volúmenes y presiones más elevados (2). En un pulmón completamente reclutado, el ciclo tidal se sitúa más proximo a la rama espiratoria de la curva y la histéresis es menor (3). 
La curva sólo explora, por lo tanto, la porción del parénquima pulmonar que se logra airear. No nos dice nada de cuánto pulmón queda sin gas en su interior, del mismo modo que no sabemos si ese volumen que entra se dirige o no a zonas que inicialmente estaban ya aireadas.

Cuando se inicia el trazado desde presión atmosférica (por lo tanto, desde capacidad residual funcional), la curva representa los límites de presión y volumen entre los cuales puede ocurrir cada ciclo ventilatorio $^{10}$. Este concepto de límite externo se representa en la figura 1B. Dentro de la curva, pueden ocurrir diferentes ciclos en función del estado de reclutamiento pulmonar al inicio y del volumen tidalpresión aplicados. En un pulmón completamente reclutado, los ciclos se sitúan próximos a la rama espiratoria de la curva, mientras que si la aireación es menor, se situarán próximos a la rama inspiratoria ${ }^{11}$. Para la ubicación exacta de un ciclo tidal, es necesario conocer el volumen teleespiratorio o al menos el volumen sobre capacidad residual funcional presente en el pulmón. Por eso la información sobre el estado del sistema respiratorio al inicio de cada ciclo tidal que nos dan las curvas presión-volumen es sólo parcial: sabemos los límites entre los que nos movemos, pero no el punto exacto de inicio de la ventilación.

\section{SIGNIFICADO DE LOS PUNTOS DE INFLEXIÓN}

La curva PV en el paciente con lesión pulmonar aguda tiene una morfología sigmoidea característica. Esto es especialmente cierto durante las fases iniciales del síndrome ${ }^{12}$. En realidad, la curva del sistema respiratorio es siempre sigmoidea, pero en las personas sanas la parte inicial de dicha curvatura ocurre a presiones subatmosféricas, que no se suelen explorar. Esta morfología sigmoidea permite identificar zonas en las cuales la curvatura (y por lo tanto la distensibilidad) cambia con mucha rapidez. A estos puntos de máxima curvatura se los ha denominado - de una manera errónea desde el punto de vista matemático pero consagrada por la literatura- puntos de inflexión ${ }^{13}$.

Se puede identificar dos puntos en la rama inspiratoria, que tradicionalmente se han denominado puntos bajo y alto de inflexión. Prácticamente todo el interés que han suscitado las curvas presión-volumen para el ajuste de la ventilación mecánica se ha centrado en estos puntos. El cambio brusco en la distensibilidad que ocurre en estas zonas refleja que «algo» está ocurriendo en el parénquima pulmonar de una manera igual de brusca. La interpretación clásica de la curva presión-volumen ${ }^{14}$ proponía que el aumento rápido de la distensibilidad y la zona de máxima distensibilidad que ocurren a presiones por encima del punto bajo de inflexión reflejan que la mayoría del parénquima pulmonar esta ya aireado, es decir, máximo reclutamiento. Por eso se propuso situar el nivel de PEEP ligeramente por encima de este punto ${ }^{15}$. De manera similar, el descenso brusco de la distensibilidad que representa el punto alto de inflexión se correspondería con el inicio de la sobredistensión al- veolar, ya que todos los alveolos, que se reclutaron a presiones mucho menores, comenzarían a estar por encima de su límite elástico. Siguiendo este razonamiento, el punto alto de inflexión marcaría la presión que no debería sobrepasarse durante la ventilación.

Sin embargo, esta interpretación ha sido desafiada. Estudios en pacientes y en animales de experimentación, con minuciosos experimentos de microscopia in vivo ${ }^{16-19}$, han demostrado que el punto bajo de inflexión representa solamente el inicio del reclutamiento. Entre presiones de cero y este punto, el fenómeno predominante es la aireación (es decir, la entrada de aire en zonas previamente aireadas), y sólo cuando la presión supera este punto comienzan a airearse zonas previamente sin gas. Cuando un alveolo no aireado se recluta (ya sea por apertura tras un colapso o por desplazamiento del líquido al intersticio, según las interpretaciones vigentes del reclutamiento), su distensibilidad aumenta bruscamente ${ }^{20}$. Teóricamente, al airearse un alveolo colapsado su distensibilidad es, en el inicio de este reclutamiento, infinita. Este es el fenómeno responsable de la zona de alta distensibilidad situada entre ambos puntos de inflexión de la rama inspiratoria.

Del mismo modo, no se ha encontrado una correlación entre el punto alto de inflexión y la sobredistensión alveolar. Aunque es difícil encontrar un patrón de referencia para el diagnóstico in vivo de la sobredistensión alveolar, se han encontrado signos de hiperaireación en pulmones lesionados desde presiones muy bajas y de manera continua a medida que aumenta la presión ${ }^{21}$. Por otro lado, sigue habiendo reclutamiento alveolar a presiones por encima del punto alto de inflexión ${ }^{22}$. Aunque se ha propuesto que este punto marca el final del reclutamiento significativo, no hay una interpretación clara de su significado. Por todo lo anterior, la interpretación actual de los puntos de inflexión de la rama inspiratoria de la curva presión-volumen pone muy en entredicho su utilidad para el ajuste de la ventilación mecánica. Las diferencias entre la interpretación clásica y la actual de los puntos de inflexión se presentan en la figura 2.

La rama espiratoria de la curva fue ignorada durante muchos años, hasta que un trabajo publicado por Hickling ${ }^{23}$ en 1998, basado exclusivamente en un modelo matemático, apoyaba su empleo para el ajuste óptimo de la PEEP. A posteriori, este planteamiento parece más razonable, ya que la PEEP es un fenómeno espiratorio y el parénquima pulmonar ya ha sido reclutado, al menos parcialmente, durante la inspiración precedente. En la rama espiratoria puede identificarse un punto de «inflexión» ${ }^{24}$, al que nos referiremos como punto de máxima curvatura para distinguirlo de los puntos de inflexión inspiratorios. Con más dificultad, en algunos pacientes se identifica un punto de inflexión inferior en la rama espiratoria. Éste es el menos estudiado, y su significado no está aclarado. Algunos autores lo han relacionado con el cierre de pequeñas vías aéreas ${ }^{25}$.

El punto de máxima curvatura marca el inicio del desreclutamiento alveolar ${ }^{16}$. Por lo tanto, una estrategia dirigida a evitar completamente el colapso alveo- 


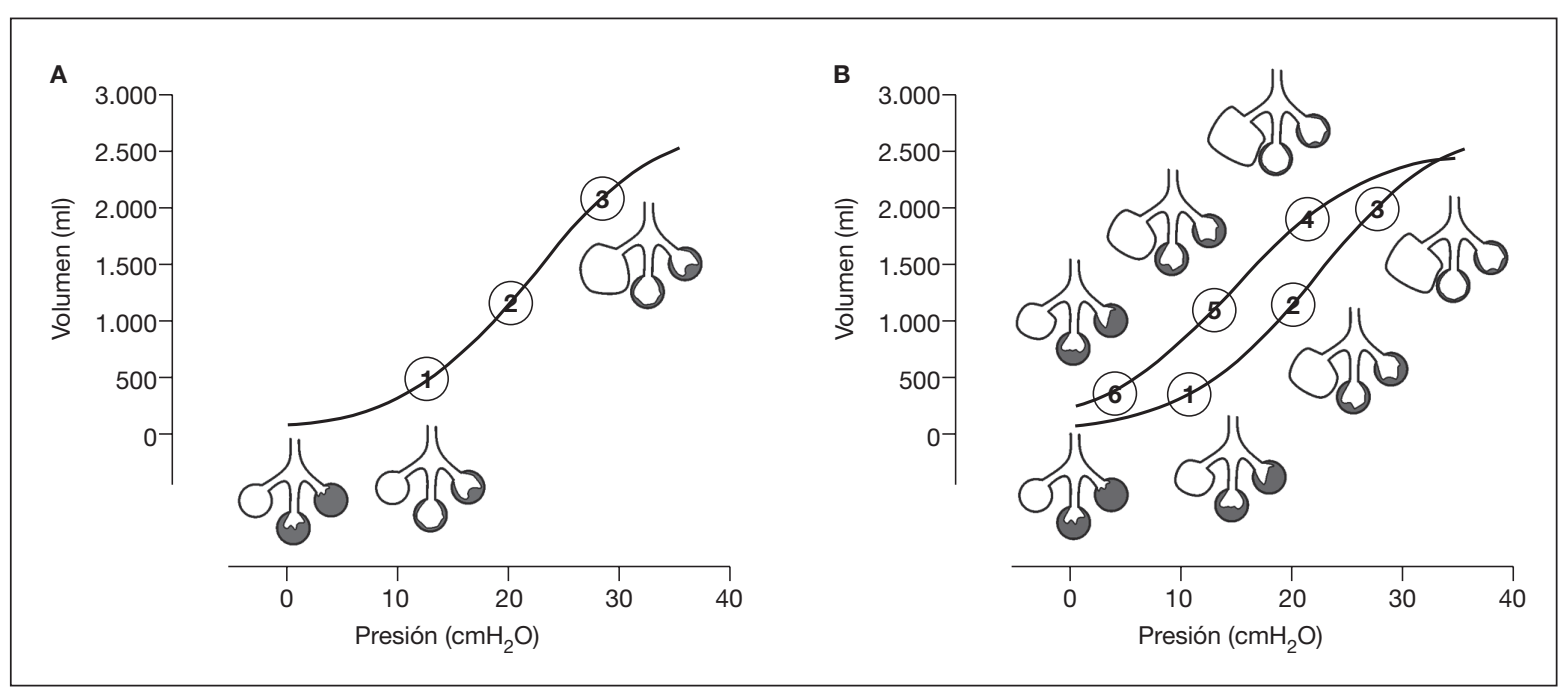

Figura 2. Diferencias entre la concepción clásica y actual de la curva presión-volumen. A: la concepción clásica presupone que el reclutamiento es casi completo a presiones situadas por encima del punto bajo de inflexión de la rama inspiratoria (1), de tal manera que el gas se reparte a los alveolos ya aireados (2), y sólo comienza la sobredistensión cuando la presión excede el punto alto de inflexión inspiratorio (3). La rama espiratoria no se tenía en cuenta. B: actualmente sabemos que el reclutamiento alveolar comienza cuando la presión sobrepasa el punto bajo de inflexión (1), y tanto reclutamiento como sobredistensión son fenómenos continuos durante toda la inspiración (2), incluso por encima del punto alto de inflexión (3). Durante la espiración, el colapso alveolar se inicia al caer la presión por debajo del punto de máxima curvatura (4) y continúa hasta el final de la espiración (5-6).

lar, como la defendida en el open lung approach, debería seleccionar este punto como nivel de PEEP. En la práctica, esto se traduce en el empleo de niveles de PEEP elevados. Los modelos animales de lesión pulmonar en los que se produce predominantemente colapso por aumento de la tensión superficial (por ejemplo, en pulmones lavados) o en animales pequeños en los que prácticamente no hay presión sobreimpuesta (es decir, la causada por el pulmón suprayacente a las zonas más dependientes del parénquima) se caracterizan por un alto potencial de reclutamiento ${ }^{26}$ y cierta resistencia al colapso, con lo que este punto de máxima curvatura se sitúa a presiones muy bajas, incluso menores que las del punto bajo de inflexión inspiratorio ${ }^{27}$. Sin embargo, en pacientes con lesión pulmonar aguda el potencial de reclutamiento es menor ${ }^{28} y$ los valores del punto de máxima curvatura se sitúan de manera casi constante a presiones por encima del punto bajo de inflexión, y algo por debajo del punto alto. En una muestra de 30 pacientes $^{29}$, los valores de los puntos bajo y alto de la rama inspiratoria y el punto de máxima curvatura espiratorio fueron de $14 \pm 4,7$, $33,2 \pm 4,1$ y $23,7 \pm 3,5 \mathrm{cmH}_{2} \mathrm{O}$, respectivamente. Esta diferencia entre los modelos y la clínica tiene importancia a la hora de interpretar los estudios experimentales y trasladar los hallazgos a la práctica, como se discute más adelante.

\section{CUESTIONES TÉCNICAS}

El trazado de curvas estáticas de presión-volumen puede ser una tarea compleja que requiere un equipamiento específico (transductores, neumotacógrafos, etc.). Recientemente, se han incorporado herramientas en el software de los ventiladores mecánicos de última generación que simplifican enormemente este proceso. Si se tiene la cautela de emplear flujos bajos en la vía aérea durante el trazado, para aproximarse lo más posible a las condiciones estáticas, diferentes métodos dan resultados superponibles. Por lo tanto, el primer escollo para la obtención de estas curvas se ha solucionado con la automatización de la maniobra.

Sin embargo, el problema del análisis de las curvas no está solucionado. La identificación de los puntos de inflexión de dichas curvas se ha realizado tradicionalmente con un método gráfico consistente en la identificación de porciones de la curva que pudiesen ajustarse mediante una línea recta, de tal manera que los puntos de inflexión se corresponderían con las intersecciones de dichas rectas ${ }^{30}$. Este método depende muchísimo del observador, y por ello está sujeto a una alta variabilidad que depende del entrenamiento de éste y de la propia disposición espacial de puntos de la curva.

Para solucionar este problema de interpretación se ha propuesto el uso de modelos matemáticos ${ }^{13}$, a los que se ajustarían los datos obtenidos durante el trazado de la curva y que permitirían el cálculo de los puntos de inflexión de una manera objetiva y reproducible. Sin embargo, los diferentes modelos propuestos resultan en diferentes valores de los puntos de inflexión para un mismo conjunto de datos ${ }^{29}$. Esta variabilidad, que es mayor para los resultados de la rama inspiratoria, probablemente se deba a que los puntos de inflexión son en realidad gamas de presión en las que ocurren cambios más o menos pronunciados en el parénquima pulmonar, y no cifras de presión concretas. De esta manera, los modelos matemáticos intentan simplificar en un valor lo que en realidad es 
una distribución completa de presiones (de aireación, de colapso, etc.).

Esta variabilidad entre observadores e incluso entre modelos hace difícil interpretar y comparar los estudios publicados. De cualquier manera, parece necesario detallar de manera precisa la metodología empleada para el análisis de las curvas en los diferentes trabajos.

Los diferentes programas incorporados en los ventiladores no incluyen herramientas de análisis más allá de cursores que pretenden facilitar la identificación de los puntos de inflexión. Posiblemente la adición de una herramienta de análisis matemático de las curvas sea el siguiente paso que dar en el diseño de software para el trazado de curvas.

\section{UTILIDAD CLÍNICA DE LAS CURVAS PRESIÓN-VOLUMEN}

La combinación de estudios clínicos con objetivos fisiopatológicos y estudios experimentales ha permitido clarificar el significado de las curvas presión-volumen en su mayor parte. Sin embargo, conocer los fenómenos que reflejan las curvas no supone que podamos deducir inmediatamente su aplicación en la práctica clínica. Se ha intentado utilizar la curva presión-volumen para la selección del nivel óptimo de PEEP, para el ajuste del volumen tidal (o presión en meseta) y para la monitorización del reclutamiento alveolar tras un cambio en la PEEP.

La aplicación estrella de las curvas presión-volumen ha sido la selección de la PEEP óptima en pacientes con lesión pulmonar aguda. Las dificultades comienzan cuando se considera el significado de «PEEP óptima»: según los autores, PEEP óptima puede hacer referencia a la que maximiza el reclutamiento alveolar ${ }^{31}$, la que se asocia a menos lesión relacionada con ventilación mecánica ${ }^{32} \mathrm{o}$ incluso a la que genera la menor alteración hemodinámica posible $^{33}$.

Prescindiendo de los aspectos hemodinámicos (no por poco importantes, sino porque su revisión excedería el objetivo del presente artículo), podríamos intentar definir una PEEP óptima como la que consigue reclutar completamente el pulmón lesionado sin producir sobredistensión en el pulmón sano. Para que esto sea posible, la distribución de las presiones de reclutamiento no debería superponerse con las presiones en las que se inicia la sobredistensión en las zonas aireadas (fig. 3A). Es posible que esto sea cierto en modelos experimentales de lesión en los que hay un importante reclutamiento a presiones bajas, como tras el lavado alveolar. Sin embargo, sabemos que en pacientes con lesión pulmonar aguda ambas distribuciones de presión abarcan una amplia gama de presiones superpuestas (fig. 3B). De este modo, no existe una «PEEP óptima», del mismo modo que no hay fármacos sin efectos secundarios. El debate sobre la PEEP se convierte en una cuestión de riesgo-beneficio, en la que las ventajas del reclutamiento (mejor gasometría, mayor estabilidad alveolar, menor colapso cíclico de alveolos) se deben sopesar frente a las desventajas de la sobredistensión (mayor espacio muerto alveolar, sobrecarga del ventrículo derecho, volutrauma $)^{34}$. Esta cuestión aún está sin resolver.

Las curvas presión-volumen nos permiten identificar, usando los puntos de inflexión, cifras de PEEP que se asocien a uno u otro objetivo, y que se resumen en la tabla 1. Como ya se comentó, el punto bajo de inflexión de la rama inspiratoria se ha utilizado tradicionalmente como PEEP óptima, aunque los datos actuales indican que el razonamiento sobre el que se basaba su empleo es erróneo. Dos ensayos clínicos ${ }^{5,6}$ han demostrado una disminución en la mortalidad en pacientes ventilados con un volumen tidal bajo y una PEEP seleccionada según este punto, al compararlo

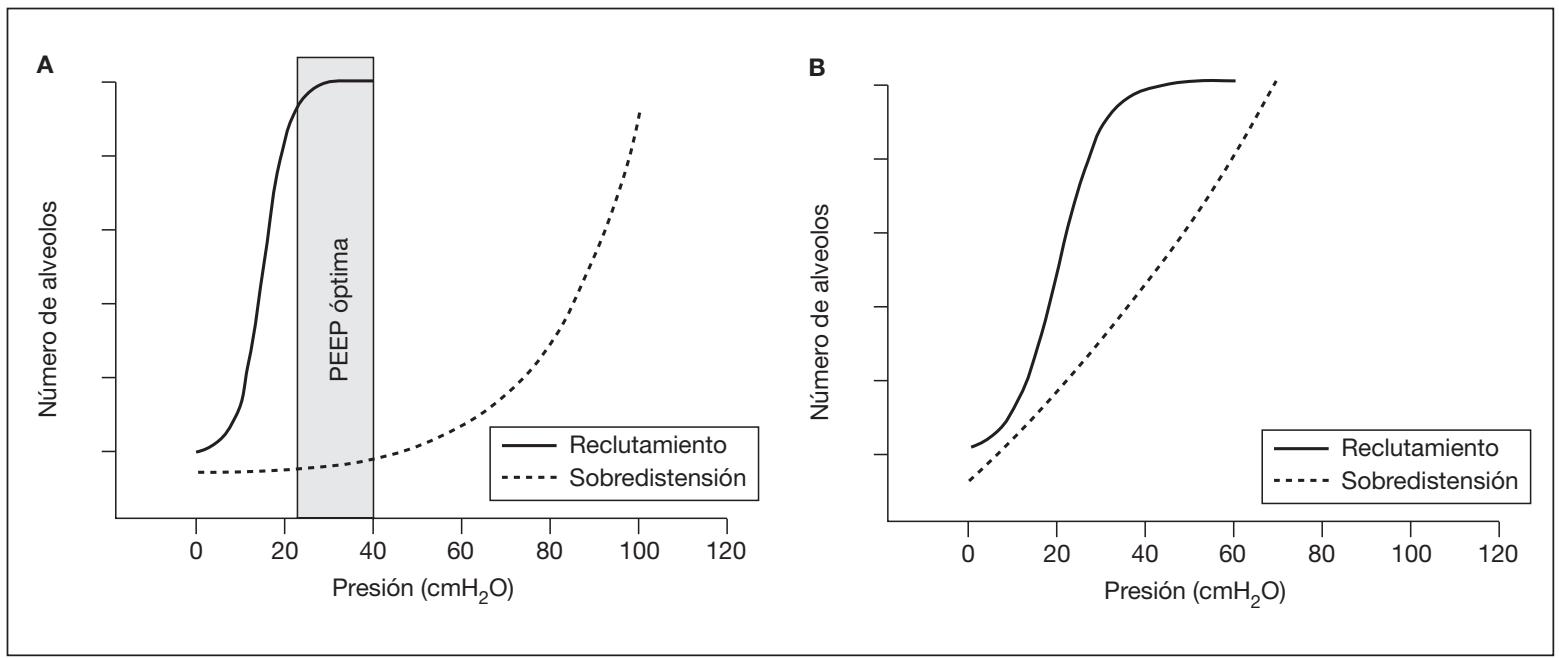

Figura 3. Distribuciones hipotéticas de las presiones de reclutamiento y sobredistensión alveolar. Si las distribuciones están lo suficientemente separadas, es posible encontrar una gama de presiones que maximizan el reclutamiento sin causar sobredistensión (A), tal y como ocurre en modelos experimentales. Por el contrario, en la lesión pulmonar aguda en pacientes, reclutamiento y sobredistensión son fenómenos superpuestos, de tal manera que no hay un nivel óptimo de PEEP (B). 
TABLA 1. Ventajas e inconvenientes de cada uno de los puntos de inflexión de la curva presión-volumen para el ajuste de la ventilación mecánica

\begin{tabular}{|l|l|c|c|c|}
\hline & Punto de inflexión & Utilidad & Ventajas & Inconvenientes \\
\hline Rama inspiratoria & Bajo & Selección de PEEP & $\begin{array}{c}\text { Asegura el inicio del } \\
\text { reclutamiento }\end{array}$ & $\begin{array}{c}\text { No explota el máximo } \\
\text { potencial de } \\
\text { reclutamiento }\end{array}$ \\
Rama espiratoria & Alto & $\begin{array}{c}\text { Selección máxima } \\
\text { presión en meseta } \\
\text { Selección de PEEP } \\
\text { de sobredistensión }\end{array}$ & $\begin{array}{c}\text { Desconocidas } \\
\text { Explota el máximo } \\
\text { potencial de } \\
\text { reclutamiento }\end{array}$ \\
\hline
\end{tabular}

con estrategias de volumen tidal elevado. Las características de este grupo control hacen difícil determinar si esta disminución de la mortalidad se debe al método seleccionado para el ajuste de PEEP, el volumen tidal bajo o una combinación de ambas estrategias. Más aún, no podemos saber si el empleo de curvas de presión-volumen es mejor que otras formas de selección de PEEP.

Las estrategias que proponen el máximo reclutamiento y la mayor estabilidad alveolar posibles deberían emplear el punto de máxima curvatura de la rama espiratoria como marcador del nivel de PEEP. Hasta la actualidad, este punto solo se ha empleado en estudios experimentales ${ }^{32}$ o estudios fisiológicos con un número de pacientes pequeño ${ }^{24,35}$, por lo que su superioridad está por demostrar. Como ya se dijo al inicio de esta sección, el problema de estas estrategias y, por lo tanto, del empleo de este punto como nivel de PEEP es que se asocia indefectiblemente a un aumento de la hiperaireación y sobredistensión de zonas sanas. Los mecanismos de lesión inducida por ventilación mecánica se inician tanto por volúmenes excesivamente bajos como por los excesivamente altos; sin embargo, no sabemos cuál es la aportación individual de cada uno de ellos. Dos ensayos clínicos ${ }^{36,37}$ recientemente publicados que comparaban valores de PEEP altos y bajos (ninguno de ellos utilizando curvas presión-volumen) fallaron en encontrar diferencias en la mortalidad, y algunos endpoints secundarios fueron favorables al grupo de PEEP alta. Aunque estos datos parecen indicar que la relación riesgo/beneficio entre la sobredistensión inducida por PEEP elevada (es seguro que en estos grupos la había) y el reclutamiento conseguido es similar a la de otras estrategias consideradas «seguras» (volúmenes bajos y PEEP bajas), es necesaria una nueva generación de estudios de laboratorio y fisiopatológicos dirigidos a comparar no ya estrategias de ventilación lesivas frente a protectoras, sino diferentes estrategias de protección pulmonar entre sí.

Las curvas presión-volumen también se han utilizado como marcador de la presión máxima en meseta que se puede alcanzar sin producir sobredistensión. Para ello se ha propuesto el punto alto de inflexión de la rama inspiratoria como marcador ${ }^{38}$. Como ya se ha discutido, la base para esta propuesta es la creencia de que la disminución de la distensibilidad que ocurre a partir de dicho punto alto de inflexión se debe al inicio de la sobredistensión alveolar. El artículo responsable de este razonamiento fue publicado por Roupie et $\mathrm{al}^{39}$ en 1995. En dicho trabajo, que ha sido citado más de 150 veces, se parte de la idea de que el punto alto de inflexión es realmente un marcador de sobredistensión, y se estudia a cuántos pacientes con lesión pulmonar aguda se ventila con presiones por encima de dicho punto. En ningún momento se asocia claramente el punto alto con la sobredistensión, por lo que se llega a un razonamiento circular. Aunque es difícil el estudio in vivo de la sobredistensión alveolar, ya que carecemos de un estándar, no se ha podido demostrar que ésta comience de manera abrupta a presiones por encima de este punto. Los estudios tomográficos han demostrado que la hiperaireación (que tampoco es un buen marcador de sobredistensión) comienza a presiones muy por debajo del punto alto de inflexión ${ }^{21,40}$. Por otro lado, se ha demostrado que aún hay reclutamiento alveolar por encima de esta gama de presiones $^{18}$, aunque en qué magnitud sea objeto de debate. Por lo tanto, no hay ninguna evidencia que respalde el empleo del punto alto de inflexión de la rama inspiratoria como marcador de sobredistensión y, por lo tanto, como guía para la selección del volumen tidal.

Una tercera utilidad para la que se han empleado las curvas presión-volumen es la cuantificación del reclutamiento después de un cambio en el nivel de PEEP. Para ello se trazan curvas desde diferentes niveles de PEEP y se compara el volumen pulmonar a una presión previamente establecida. El desplazamiento hacia arriba de la curva se interpreta como reclutamiento inducido por la PEEP. Este concepto se introdujo en $1994^{41} \mathrm{y}$ asume que el aumento de volumen se debe exclusivamente al reclutamiento. Sin embargo, estudios por tomografía computarizada (TC) han mostrado de manera uniforme que, a medida que aumenta la presión en la vía aérea, el aumento de volumen se distribuye entre lo que se ha definido como aireación (entrada de aire en zonas previamente aireadas) y reclutamiento ${ }^{28,42,43}$. De esta manera, el resultado esperable, y demostrado en $2006^{44}$, es que la curva presión-volumen sobrestima el reclutamiento y que, aunque la correlación entre el volumen reclutado medido por TC o usando curvas es significativa, los límites de acuerdo son muy amplios, por lo que no es aplicable en casos individuales.

La predicción del potencial de reclutamiento de un paciente con lesión pulmonar aguda podría ser de gran importancia; los pacientes con alto potencial de reclutamiento tienen mayor gravedad y mortalidad que los 
que no lo tienen, y probablemente el ajuste de la ventilación mecánica debería ser diferente ${ }^{45}$. Aunque las curvas presión-volumen no sean exactas en la cuantificación de dicho reclutamiento, es posible que alguna característica de dichas curvas permitiría la predicción de dicho potencial. En una publicación reciente, la histéresis de la curva permitió predecir la respuesta a una maniobra de reclutamiento en un modelo animal de lesión pulmonar ${ }^{46}$. Está por ver si este tipo de medidas son eficaces en pacientes.

\section{CONCLUSIONES}

Las curvas estáticas de presión-volumen son una herramienta excelente para aprender fisiología respiratoria. En pacientes con lesión pulmonar aguda, además permiten identificar la gama de presiones en que ocurren sucesos relevantes como el inicio del reclutamiento o del colapso alveolar. Hay amplia evidencia de estudios experimentales y en pacientes sobre esta relación de los puntos de inflexión con los cambios en la aireación del parénquima.

Sin embargo, la aplicación clínica de este conocimiento está menos aclarada. Aunque se ha propuesto el uso de dichas curvas para la selección de PEEP en pacientes con lesión pulmonar, no hay datos robustos que respalden este método frente a otros más sencillos. Más aún, la comprensión de los mecanismos de reclutamiento, aireación y pérdida de la aireación alveolar, a la que tanto han contribuido estas curvas, indica que no hay un punto óptimo de PEEP en este grupo de pacientes, y que la selección de esta presión debe balancearse entre las ventajas del reclutamiento y los inconvenientes de la sobredistensión. No disponemos actualmente de conocimiento que incline esta balanza en un sentido u otro.

Por lo tanto, no se puede recomendar el empleo sistemático de estas curvas para el ajuste de la ventilación en pacientes con lesión pulmonar aguda. Es todo un dilema decidir si la investigación debería dirigirse a la búsqueda de una utilidad para este tipo de medidas o hacia otros métodos para el ajuste de la ventilación mecánica.

\section{BIBLIOGRAFÍA}

1. Fernández Fernández R. [Pathophysiology of gas exchange in ARDS]. Med Intensiva 2006;30:374-8.

2. Gordo Vidal F, Delgado Arnaiz C, Calvo Herranz E. [Mechanical ventilation induced lung injury]. Med Intensiva. 2007:31:18-26.

3. Pedreira PR, Garcia-Prieto E, Albaiceta GM, Taboada F. [Inflammatory response and apoptosis in acute pulmonary injury]. Med Intensiva. 2006;30:268-75

4. Ventilation with lower tidal volumes as compared with traditional tidal volumes for acute lung injury and the acute respiratory distress syndrome. The Acute Respiratory Distress Syndrome Network. N Engl J Med. 2000;342:1301-8.

5. Amato MB, Barbas CS, Medeiros DM, Magaldi RB, Schettino GP, Lorenzi-Filho G, et al. Effect of a protective-ventilation strategy on mortality in the acute respiratory distress syndrome. N Engl J Med. 1998:338:347-54

6. Villar J, Kacmarek RM, Perez-Mendez L, Aguirre-Jaime A. A high positive end-expiratory pressure, low tidal volume ventilatory strategy improves outcome in persistent acute respiratory distress syndrome: a randomized, controlled trial. Crit Care Med. 2006;34: $1311-8$.

7. Ventrice EA, Marti-Sistac O, Gonzalvo R, Villagra A, LopezAguilar J, Blanch L. [Molecular and biophysical mechanisms and modulation of ventilator-induced lung injury]. Med Intensiva. 2007;31:73-82.

8. Gattinoni L, Carlesso E, Cadringher P, Valenza F, Vagginelli F, Chiumello D. Physical and biological triggers of ventilator-induced lung injury and its prevention. Eur Respir J Suppl. 2003;47: S15-25.

9. Valenza F, Guglielmi M, Maffioletti M, Tedesco C, Maccagni $\mathrm{P}$, Fossali T, et al. Prone position delays the progression of ventilator-induced lung injury in rats: does lung strain distribution play a role? Crit Care Med. 2005;33:361-7.

10. Rimensberger PC, Pristine G, Mullen BM, Cox PN, Slutsky AS. Lung recruitment during small tidal volume ventilation allows minimal positive end-expiratory pressure without augmenting lung injury. Crit Care Med. 1999;27:1940-5.

11. Downie JM, Nam AJ, Simon BA. Pressure-volume curve does not predict steady-state lung volume in canine lavage lung injury. Am J Respir Crit Care Med. 2004;169:957-62.

12. Matamis D, Lemaire F, Harf A, Brun-Buisson C, Ansquer JC, Atlan G. Total respiratory pressure-volume curves in the adult respiratory distress syndrome. Chest. 1984;86:58-66.

13. Venegas JG, Harris RS, Simon BA. A comprehensive equation for the pulmonary pressure-volume curve. J Appl Physiol. 1998;84:389-95

14. Putensen C, Baum M, Hormann C. Selecting ventilator settings according to variables derived from the quasi-static pressure/ volume relationship in patients with acute lung injury. Anesth Analg. 1993;77:436-47.

15. Mergoni M, Volpi A, Bricchi C, Rossi A. Lower inflection point and recruitment with PEEP in ventilated patients with acute respiratory failure. J Appl Physiol. 2001;91:441-50.

16. Albaiceta GM, Taboada F, Parra D, Luyando LH, Calvo J, Menendez R, et al. Tomographic study of the inflection points of the pressure-volume curve in acute lung injury. Am J Respir Crit Care Med. 2004;170:1066-72.

17. Jonson B, Richard JC, Straus C, Mancebo J, Lemaire F, Brochard L. Pressure-volume curves and compliance in acute lung injury: evidence of recruitment above the lower inflection point. Am J Respir Crit Care Med. 1999;159:1172-8.

18. Maggiore SM, Jonson B, Richard JC, Jaber S, Lemaire F, Brochard L. Alveolar derecruitment at decremental positive endexpiratory pressure levels in acute lung injury. Comparison with the lower inflection point, oxygenation, and compliance. Am J Respir Crit Care Med. 2001;164:795-801.

19. DiRocco JD, Carney DE, Nieman GF. Correlation between alveolar recruitment/derecruitment and inflection points on the pressure-volume curve. Intensive Care Med. 2007;33: 1204-11.

20. Dambrosio M, Roupie E, Mollet JJ, Anglade MC, Vasile N, Lemaire $\mathrm{F}$, et al. Effects of positive end-expiratory pressure and different tidal volumes on alveolar recruitment and hyperinflation. Anesthesiology. 1997;87:495-503.

21. Vieira SR, Puybasset L, Richecoeur J, Lu Q, Cluzel P, Gusman PB, et al. A lung computed tomographic assessment of positive end-expiratory pressure-induced lung overdistension. Am J Respir Crit Care Med. 1998;158:1571-7.

22. Borges JB, Okamoto VN, Matos GF, Caramez MP, Arantes PR, Barros F, et al. Reversibility of lung collapse and hypoxemia in early acute respiratory distress syndrome. Am J Respir Crit Care Med. 2006;174:268-78.

23. Hickling KG. The pressure-volume curve is greatly modified by recruitment. A mathematical model of ARDS lungs. Am J Respir Crit Care Med. 1998;158:194-202.

24. Holzapfel L, Robert D, Perrin F, Blanc PL, Palmier B, Guerin C. Static pressure-volume curves and effect of positive endexpiratory pressure on gas exchange in adult respiratory distress syndrome. Crit Care Med. 1983;11:591-7.

25. Bayle F, Guerin C, Debord S, Badet M, Lemasson S, Poupelin JC, et al. Assessment of airway closure from deflation lung volume-pressure curve: sigmoidal equation revisited. Intensive Care Med. 2006;32:894-8. 
26. Pelosi P, Goldner M, McKibben A, Adams A, Eccher G, Caironi $\mathrm{P}$, et al. Recruitment and derecruitment during acute respiratory failure: an experimental study. Am J Respir Crit Care Med. 2001;164:122-30.

27. Rimensberger PC, Cox PN, Frndova H, Bryan AC. The open lung during small tidal volume ventilation: concepts of recruitment and "optimal" positive end-expiratory pressure. Crit Care Med. 1999;27:1946-52.

28. Crotti S, Mascheroni D, Caironi P, Pelosi P, Ronzoni G, Mondino M, et al. Recruitment and derecruitment during acute respiratory failure: a clinical study. Am J Respir Crit Care Med. 2001;164:131-40.

29. Albaiceta GM, Garcia E, Taboada F. Comparative study of four sigmoid models of pressure-volume curve in acute lung injury. Biomed Eng Online. 2007;6:7.

30. Harris RS, Hess DR, Venegas JG. An objective analysis of the pressure-volume curve in the acute respiratory distress syndrome. Am J Respir Crit Care Med. 2000;161:432-9.

31. Lachmann B. Open up the lung and keep the lung open. Intensive Care Med. 1992;18:319-21.

32. Takeuchi M, Goddon S, Dolhnikoff M, Shimaoka M, Hess $\mathrm{D}$, Amato MB, et al. Set positive end-expiratory pressure during protective ventilation affects lung injury. Anesthesiology. 2002;97:682-92.

33. Page B, Vieillard-Baron A, Beauchet A, Aegerter P, Prin S, Jardin F. Low stretch ventilation strategy in acute respiratory distress syndrome: eight years of clinical experience in a single center. Crit Care Med. 2003;31:765-9.

34. Albaiceta GM, Luyando LH, Parra D, Menendez R, Calvo J, Pedreira PR, et al. Inspiratory vs. expiratory pressure-volume curves to set end-expiratory pressure in acute lung injury. Intensive Care Med. 2005;31:1370-8.

35. Albaiceta GM, Luyando LH, Parra D, Menéndez R, Calvo J, Taboada F. Inspiratory vs expiratory limb of the pressure-volume curve for the positive end-expiratory pressure setting in acute lung injury. Crit Care. 2003;8 Suppl 1:25.

36. Meade MO, Cook DJ, Guyatt GH, Slutsky AS, Arabi YM, Cooper DJ, et al. Ventilation strategy using low tidal volumes, recruitment maneuvers, and high positive end-expiratory pressure for acute lung injury and acute respiratory distress syndrome: a randomized controlled trial. JAMA. 2008;299:637-45.
37. Mercat A, Richard JC, Vielle B, Jaber S, Osman D, Diehl JL, et al. Positive end-expiratory pressure setting in adults with acute lung injury and acute respiratory distress syndrome: a randomized controlled trial. JAMA. 2008;299:646-55.

38. Servillo G, De Robertis E, Maggiore S, Lemaire F, Brochard $\mathrm{L}$, Tufano R. The upper inflection point of the pressure-volume curve. Influence of methodology and of different modes of ventilation. Intensive Care Med. 2002;28:842-9.

39. Roupie E, Dambrosio M, Servillo G, Mentec H, El Atrous S, Beydon L, et al. Titration of tidal volume and induced hypercapnia in acute respiratory distress syndrome. Am J Respir Crit Care Med. $1995 ; 152: 121-8$.

40. Nieszkowska A, Lu Q, Vieira S, Elman M, Fetita C, Rouby JJ. Incidence and regional distribution of lung overinflation during mechanical ventilation with positive end-expiratory pressure. Crit Care Med. 2004;32:1496-503.

41. Ranieri VM, Giuliani R, Fiore T, Dambrosio M, Milic-Emili J. Volume-pressure curve of the respiratory system predicts effects of PEEP in ARDS: "occlusion" versus "constant flow" technique. Am J Respir Crit Care Med. 1994;149:19-27.

42. Gattinoni L, Pesenti A, Avalli L, Rossi F, Bombino M. Pressure-volume curve of total respiratory system in acute respiratory failure. Computed tomographic scan study. Am Rev Respir Dis. 1987; 136:730-6.

43. Rouby JJ, Puybasset L, Cluzel P, Richecoeur J, Lu Q, Grenier P. Regional distribution of gas and tissue in acute respiratory distress syndrome. II. Physiological correlations and definition of an ARDS Severity Score. CT Scan ARDS Study Group. Intensive Care Med. 2000;26:1046-56.

44. Lu Q, Constantin JM, Nieszkowska A, Elman M, Vieira S, Rouby JJ. Measurement of alveolar derecruitment in patients with acute lung injury: computerized tomography versus pressure-volume curve. Crit Care. 2006;10:R95.

45. Gattinoni L, Caironi P, Cressoni M, Chiumello D, Ranieri VM, Quintel M, et al. Lung recruitment in patients with the acute respiratory distress syndrome. N Engl J Med. 2006;354: 1775-86.

46. Koefoed-Nielsen J, Nielsen ND, Kjaergaard AJ, Larsson A. Alveolar recruitment can be predicted from airway pressure-lung volume loops: an experimental study in a porcine acute lung injury model. Crit Care. 2008;12:R7. 